\title{
A Simulation on the Improved Energy Saving Protocol of AODV for Mobile Ad Hoc Network
}

\author{
Fengying $\mathrm{Xu}^{\mathrm{a}}$, Zhimin Liu ${ }^{\mathrm{a},{ }^{*}}$, Yongchun $\mathrm{Xu}^{\mathrm{b}}$ \\ ${ }^{a}$ Key Laboratory of Key Technology on Agricultural Machine and Equipment (South China Agricultural \\ University), Ministry of Education, Guangzhou 510642, China \\ ${ }^{b}$ Department of Computer Technology, Guangdong Institute of Science and Technology, Guangzhou 510091,
} China

\begin{abstract}
The fixed communication interruption easily occurs in extreme disaster situation, so it needs a robust mobile Ad hoc wireless communication to deliver the rescue information with high quality and efficiency. But the widespread excessive energy consumptions loaded in mobile Ad hoc wireless routing network have become the restriction bottleneck of its application. Based on the routing protocol of AODV, this paper proposed a routing algorithm of AODVSE energy-saving protocol, and studied its effects of the working performance on mobile Ad hoc wireless network. The simulation results showed that the AODVSE protocol could be better to improve the energy-saving performance than AODV and DSR protocols by reducing the routing expiration time and the routing load and extending the network lifetime, in order to help to implement the accurate and immediate rescue.
\end{abstract}

Index Terms: Ad Hoc Network; Routing Algorithm; Energy-saving Routing; NS2 Simulation

(C) 2011 Published by MECS Publisher. Selection and/or peer review under responsibility of the International Conference on E-Business System and Education Technology

\section{Introduction}

In recent years, as extreme disastrous weather suddenly and frequently happens, it often causes huge casualties and the fixed communication interruption, so there need an robust mobile Ad hoc wireless network to deliver the rescue information. At present, in mobile Ad hoc wireless network, the huge requirements of massive communication are increasing, but its wireless network routing connection channels are increasingly restricted by their physical characteristics, which has caused the competent of neighboring data flow channels, and affected the allocation of resources and the working performance of nodes [1-3]. Recently, aimed to the research on mobile Ad hoc wireless network routing protocol under massive information, the simulation tests have the unique characteristics of scientific, reliability, efficiency and low cost, which have been employed by various research institutions, but the widespread excessive energy consumptions existing in mobile Ad hoc

* Corresponding author:

E-mail address: army258@163.com 
wireless routing protocol are loaded on this network, which has become the restriction bottleneck of its civil and military application [4-5].

To help to implement the accurate and immediate rescue and solve the excessive energy consumptions in the mobile Ad hoc wireless network, building a test platform of the energy-saving performance has an important theoretical and practical significance in the application of disaster relief. Currently, the mobile Ad hoc wireless network energy-saving routing protocol mainly has two design methods [6]: minimize the energy consumption of every packet delivery and maximize the network lifetime of the nodes with small residual energy.

Although minimizing the energy of every packet delivery could reduce the consumption in routing process, the residual energy of nodes was neglected, which may lead to some of the key nodes being frequently used and network faults. Since the minimal routing cost is ignored, and the death of some key nodes cannot be avoided because of the use of excessive energy, the similar problems of the energy-saving performance appeared in the method of maximizing the network lifetime, which can also result in network faults. Therefore, this paper would launch a research on the energy-saving routing protocol for mobile Ad hoc wireless network in the disaster environment, in order to ensure its communication quality and to increase the reliability and efficiency of rescue under massive information.

\section{Design of Energy-Saving AODV Routing Protocol for Disaster Situation}

Based on AODV (Ad hoc On demand Distance Vector) [7] routing protocol, this paper proposed a kind of energy-saving routing algorithm AODVSE (AODV based on Saving Energy). The main idea of AODVSE routing protocol was that not only reducing the times of each packet delivery [8] and the routing expiration time to lower the routing load, but also increasing the delay to reduce energy consumption, so as to achieve the purpose of extending network lifetime.

In the routing discovery process, the nodes for massive information in disaster area obtained the fewer routing information, AODV routing protocol constantly executed this process. Meanwhile, mobile Ad hoc wireless network topology constantly changed, so the routing information need to be updated constantly, resulting in the multiple retransmissions of RREQ (Routing REQuirement). Based on NS2 software simulation platform, this paper set two variables RREQ_RETRIES and TTL_START to 3 and 2 in the file Aodv.h for AODV routing protocol, and modified ACTIVE_ROUTE_TIMEOUT to 5 so as to reduce the routing expiration time, the detailed setting as follows:

\section{\# Define RREQ_RETRIES 2}

\# Define ACTIVE_ROUTE_TIMEOUT 5

\# Define TTL_START 2

The above design of the routing protocol AODVSE was proposed based on the routing improvement of AODV protocol in disaster area for saving energy under massive information, considering every node which would received the RREQ, when they received the RREQ, according to their residual energy of RemEnergy and initial energy of RemEnergy, the routing delay weights of DelayWeight and the corresponding delay functions of DelayEnergy could be obtained.

\section{NS2 Simulation Environment of the Mobile Ad hoc Wireless Networks}

Based on the design of mobile Ad hoc wireless network routing protocol ADOVSE for disaster situation communication, this paper used the free software NS2 (Network Simulator Version2) with a scalable discrete event network simulation function and an open structure, added new elements on the level of NS2 source code database with C++ and Otcl, through modifying the NS2 itself source code to realize a complete network communication simulation platform. Fig. 1 showed the flowchart of entire simulation process. 


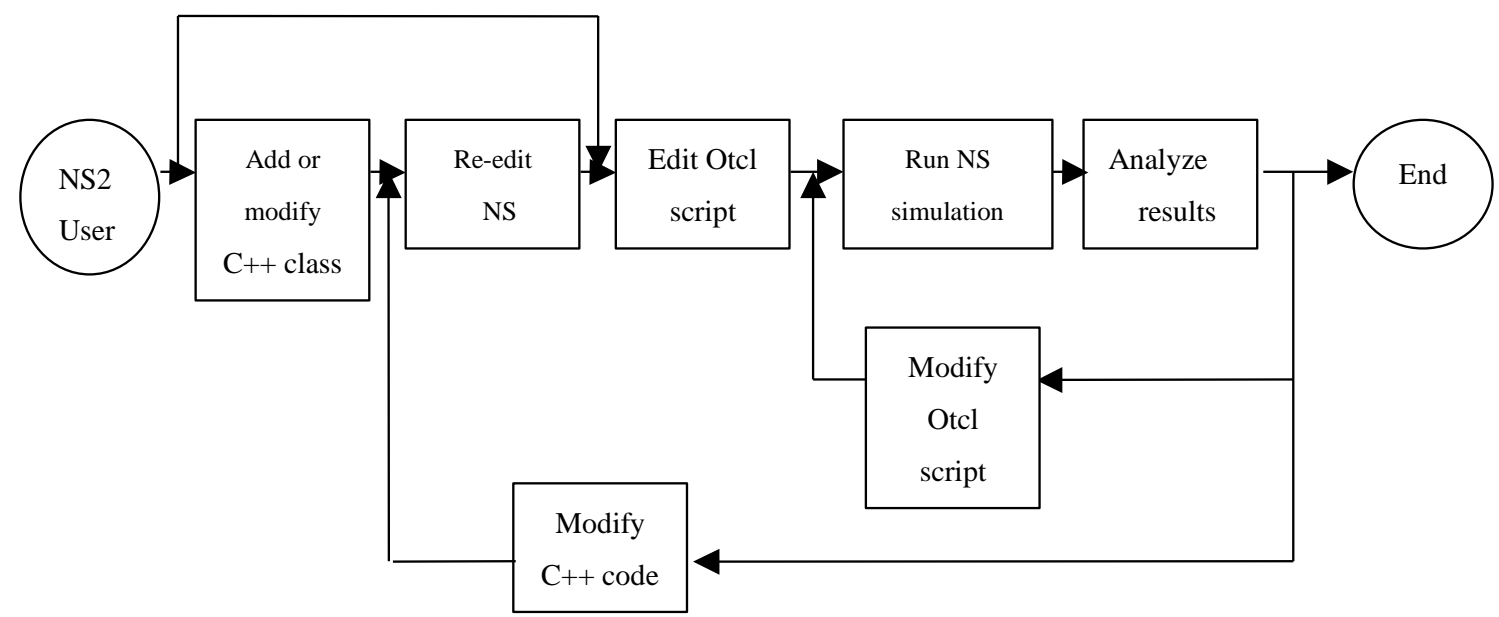

Fig. 1. Flowchart of the AODV, AODVSE and DSR routing protocols simulation

Through NS2 network simulation software, the routing simulation of AODVSE protocol was done. The scenes of random data flow and movement were set as: the network topology of $1000 \mathrm{~m} * 300 \mathrm{~m}$, the movement nodes of 50, the residence time of $0 \mathrm{~s}$, the maximum node moving speed of $20 \mathrm{~ms}^{-1}$, the simulation time of $300 \mathrm{~s}$, the MCNN (Maximum Connection Number of Nodes) of 10,15,20,25,30,35,40, the node initial energy of 200J, the node output power of $1.327 \mathrm{~W}$, the received power of $0.866 \mathrm{~W}$. The MAC layer protocol of IEEE802.11 was used, and Two-Ray Ground Reflection Model was set as the wireless transmission model, whose carrier frequency was $914 \mathrm{MHz}$ and bandwidth was $2 \mathrm{Mbps}$ with an Omni Antenna. In the simulation of AODV, AODVSE, DSR protocols, GAWK and GNUPLOT were used to obtain the analysis diagrams of AEED (Average End-to-End Delay), PDR (Packet Delivery Ratio), and SN (Survival Nodes) [9].

\section{Results Analysis of AODV, AODVSE and DSR Routing Protocols Simulation}

Through above simulation, Fig. 2 to Fig. 4 showed the contrast results of AEED, PDR and SN in three routing protocols AODV, DSR (Dynamic Source Routing) [10] and AODVSE, respectively.

In Fig. 2, it could be seen when the MCNN was less than 20, the AEED of three protocols AODV, DSR and AODVSE were very small, almost equaled to 0. If the MCNN was greater than 20, the AEED of routing protocols AODV and AODVSE increased slowly, and the AEED of routing protocol AODVSE increased slightly larger than that of AODV, which accorded with increasing the delay of routing protocol AODVSE through the time-delay function, but the AEED of DSR protocol appeared more dramatic increase and larger than that of routing protocols AODV and AODVSE. 


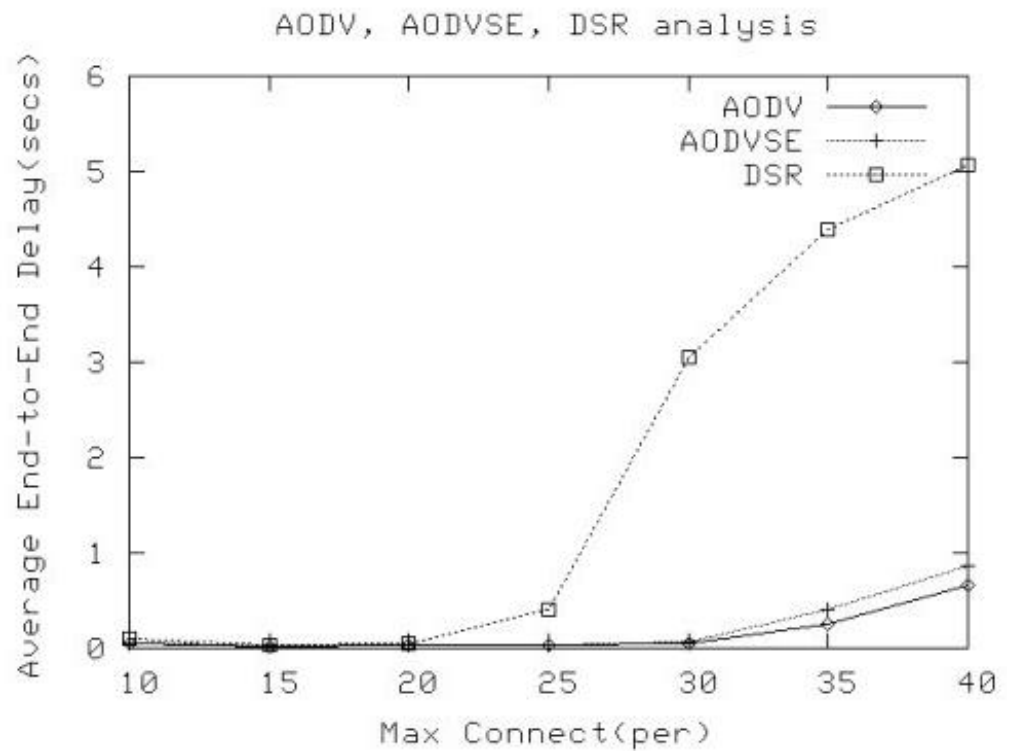

Fig. 2. Contrast of the average end-to-end delay

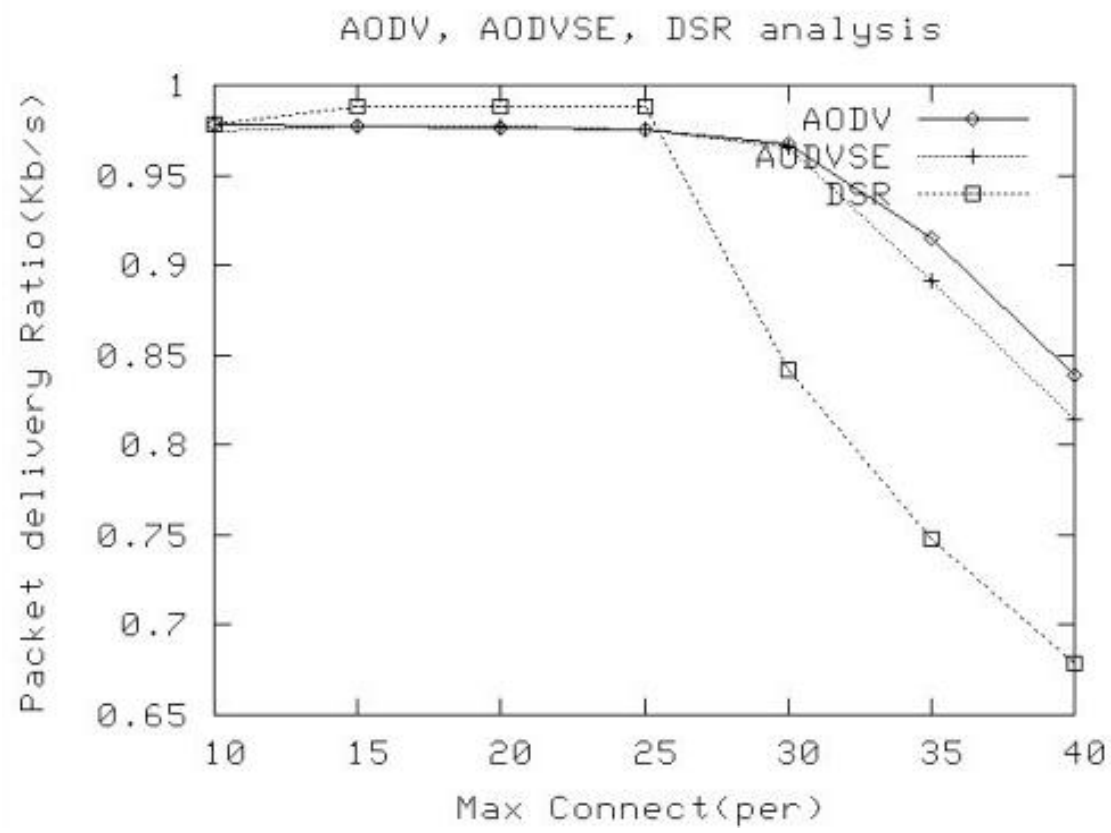

Fig. 3. Contrast of the packet delivery ratio

In Fig. 3, when the MCNN was less than 25, the PDR of three protocols AODV, DSR and AODVSE were little difference. The PDR of routing protocol DSR was slightly better than that of AODV and AODVSE, 
almost reached $100 \%$. But when the MCNN was greater than 25, the PDR of three protocols AODV, DSR and AODVSE declined sharply, and the PDR of DSR was significantly lower than that of AODV and AODVSE. As routing protocol AODVSE reduced the packet delivery times and the routing expiration time, the PDR of routing protocol AODVSE would be slightly smaller than that of AODV, which was consistent with the simulation results.

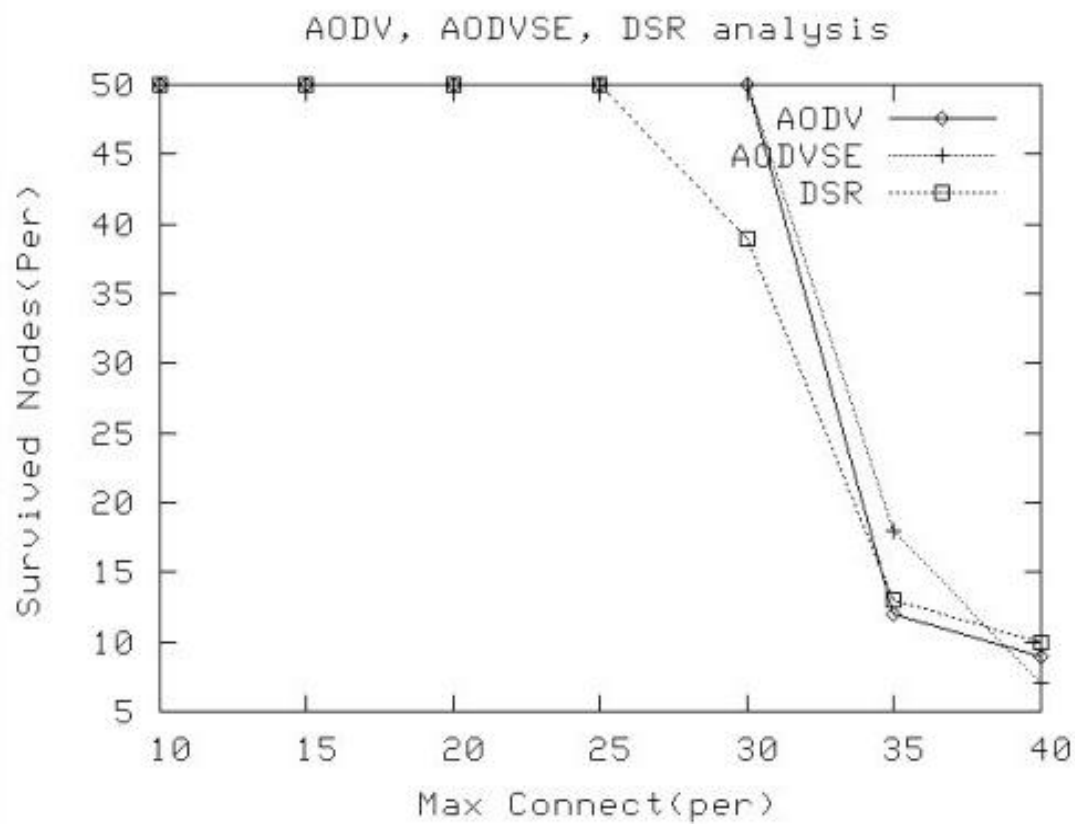

Fig. 4. Contrast of the survivial nodes

In Fig. 4, when the MCNN were greater than 30, it could be seen that the SN of routing protocol AODVSE was significantly greater than that of AODV. The reason could be that the energy-saving mechanism was used to change the delay and the times of packet delivery. But if the MCNN was larger than 30, the SN of AODVSE were more than that of AODV and DSR, which indicated that there was relatively longer network lifetime in it.

\section{Conclusions}

Mobile Ad hoc wireless network can play an important communication rescue role in the disastrous environment, it can used to replace the fixed communications network that was destroyed and to achieve the fast networking, thus, which will have an important application aspects in the field of civil and military mobile communications. The routing of mobile Ad hoc wireless network under a huge demand for massive communication has an effect on the allocation of resources and the working performance of its nodes, in the existing mobile Ad hoc on-demand wireless network routing protocols, each routing algorithm has its own advantages and disadvantages. The routing algorithm of AODVSE protocol under massive network communication has the advantages of saving energy and good working performance, which could be efficiently solve the various security problems existing in wireless network routing, to realize the communication quality under massive information by reducing excessive consumption energy. But under the communication of massive information, there are still some questions such as clustering algorithm, energy more efficiently using etc., which need to be further studied, so as to extend the life of nodes and entire network. 


\section{Acknowledgements}

This study was supported by a grant from the Natural Science Foundation of Guangdong Province, China (No.845106420100873) and the National Natural Science Foundation of China (No.31071583).

\section{References}

[1] Yih-Chun Hu, David B. Johnson, Adrian Perrig .SEAD: secure efficient distance vector routing for mobile wireless Ad hoc networks. Ad hoc Networks, Volume 1, Issue 1, July 2003, pp. 175-192

[2] Siddhartha Gupte, Mukesh Singhal. Secure routing in mobile wireless Ad hoc networks .Ad hoc Networks, Volume 1, Issue 1, July 2003, pp. 151-174

[3] Javad Akbari Torkestani, Mohammad Reza Meybodi.Mobility-based multicast routing algorithm for wireless mobile Ad-hoc networks: A learning automata approach. Computer Communications, Volume 33, Issue 6, 15 April 2010, pp. 721-735

[4] Yao Yu, Lei Guo, Xingwei Wang, Cuixiang Liur. Routing security scheme based on reputation evaluation in hierarchical Ad hoc networks . Computer Networks, Volume 54, Issue 9, 17 June 2010, pp. 1460-1469

[5] Elizabeth M. Belding-Royer, Charles E. Perkins. Evolution and future directions of the Ad hoc ondemand distance-vector routing protocol. Ad hoc Networks, Volume 1, Issue 1, July 2003, pp. 125-150

[6] WANG Shao-qing,Nie Jing-nan.Enhanced AODV routing protocol based on energy efficient.Computer Engineering and Applications, Volume 43, Issue28, 2007, pp.150-152

[7] Elizabeth M. Belding-Royer, Charles E. Perkins. Evolution and future directions of the Ad hoc ondemand distance-vector routing protocol. Ad hoc Networks, Volume 1, Issue 1, July 2003, pp. 125-150

[8] 2008 Journal of the Graduate Sun Yat-Sen University (Natura L Sciences、Medicine) vol. 29, Issue 1,Jua Issue, January 2008, pp. 117-127

[9] Li Layuan, Li Chunlin, Yaun Peiyan .Performance evaluation and simulations of routing protocols in Ad hoc networks .Computer Communications, Volume 30, Issue 8, 8 June 2007, pp. 1890-1898

[10] David B. Johnson David A. Maltz Josh Broch. DSR:Dynamic Source Routing in Ad hoc Wireless Networks. Mobile Computing. The International Series in Engineering and Computer Science, 1996, Volume 353, pp. 153-181 